\title{
In silico elucidation of potential drug target sites of the Thumb Index Fold Protein, Wnt-8b
}

\author{
Sheikh Arslan Sehgal ${ }^{1-3 *}$, Sonia Kanwal ${ }^{2}$, Rana Adnan Tahir $^{2,4}$, Zunera Khalid ${ }^{2}$, \\ Mirza A Hammad ${ }^{3,5}$ \\ ${ }^{1}$ State Key Laboratory of Membrane Biology, Institute of Zoology, Chinese Academy of Sciences, Beijing, China, ${ }^{2}$ Department \\ of Biosciences, COMSATS Institute of Information Technology, Sahiwal, Pakistan, ${ }^{3}$ University of Chinese Academy of \\ Sciences, Beijing, China, ${ }^{4}$ Beijing Key Laboratory of Separation and Analysis in Biomedical and Pharmaceuticals, Department \\ of Biomedical Engineering, School of Life Sciences, Beijing Institute of Technology, ${ }^{5}$ National Laboratory of \\ Biomacromolecules, Institute of Biophysics, Chinese Academy of Sciences, Beijing, China
}

*For correspondence: Email: arslansehgal@yahoo.com

Sent for review:

Revised accepted: 20 January 2018

\begin{abstract}
Purpose: The involvement of Wnt-8b in Wnt signaling pathway leads to various cancers. The purpose of this study was to determine the therapeutic compounds from the available library by targeting Wnt-8b using molecular docking analyses.

Methods: Threading and comparative modeling approaches were employed to predict the $3 D$ structure of Wnt-8b. Sixty-eight models were evaluated using molprobity, ERRAT and rampage evaluation tools and the model having $82.456 \%$ overall quality value was selected for further analyses. The acyl group was added to the suitable model to satisfy the hydrophobic nature of the Wnt-8b. Literature-derived compounds were selected for comparative molecular docking studies using GOLD, AutoDock and AutoDock Vina. Furthermore, docked complexes were analyzed and visualized using Chimera and Ligplot.

Results: The compound ZINC04029462 exhibited high binding potential with Wnt-8b and palmitoleic acid and was found common among top 20 compounds of each tool. His-183, Val-185, Ser-186, Gly187, Ser-188 and Thr-190 residues commonly interacted with compounds and palmitoleic acid and considered as potential interacting residues.

Conclusion: Common interacting residues from top 20 compounds of each tool suggest that these compounds may be utilized to inhibit aberrant expression of Wnt-8b. The common inhibitor ZINC04029462 may act as a lead compound for further drug designing against Wnt family.
\end{abstract}

Keywords: Wnt-8b, Cancer, Homology modeling, Molecular docking, AutoDock

\begin{abstract}
This is an Open Access article that uses a funding model which does not charge readers or their institutions for access and distributed under the terms of the Creative Commons Attribution License (http://creativecommons.org/licenses/by/4.0) and the Budapest Open Access Initiative (http://www.budapestopenaccessinitiative.org/read), which permit unrestricted use, distribution, and reproduction in any medium, provided the original work is properly credited.

Tropical Journal of Pharmaceutical Research is indexed by Science Citation Index (SciSearch), Scopus, International Pharmaceutical Abstract, Chemical Abstracts, Embase, Index Copernicus, EBSCO, African Index Medicus, JournalSeek, Journal Citation Reports/Science Edition, Directory of Open Access Journals (DOAJ), African Journal Online, Bioline International, Open-J-Gate and Pharmacy Abstracts
\end{abstract}

\section{INTRODUCTION}

The wnt proteins are highly hydrophobic in nature and addition of palmitoleic acid (PAM) increases the hydrophobicity index. The wnt proteins are difficult to isolate and crystallize due to the highly hydrophobic and subtle nature. In 2012, the first crystal structure of XWnt-8 (wnt family member) was reported [1]. PAM is a saturated fatty acid that acylates more than 100 
proteins. The PAM modification in protein showed pleiotropic effects on the functions of the proteins. Wnt-8b belongs to Wnt family of secreted proteins and has 71, 86, and 95\% sequence identity to Xenopus, zebrafish and mouse respectively [2]. Wnt-8b is located at chromosomal location 10q24 having the sequence length of 351 residues. First 22 amino acids behave as the signal peptide and remaining 329 residues are part of the mature peptide.

The wnt signaling is considered as an essential mechanism in cell fate determination, cell polarity and cell proliferation during tissue homeostasis and embryonic development [3]. The wnt pathway was characterized primarily for its developmental role and dysregulation of wnt signal transduction leads to the development of tumor in humans. $\beta$-catenin is a regulator of wnt signaling and the level of the cytoplasmic pool of $\beta$-catenin increases by the gene mutation in axin or the binding of wnt ligands with frizzled receptors. The accumulation of $\beta$-catenin in cytoplasm increases the $\beta$-catenin binding to Lef/Tcf transcription factors. $\beta$-catenin binding led to increasing in the transcriptional activity of the proteins (c-Myc and cyclin D1) which controls $\mathrm{G} 1$ phase to $S$ phase in the cell cycle [4]. The mutation detected in exon 3 of $\beta$-catenin causes disruption in wnt signaling pathway and leads to gastric cancer [5]. In pancreatic cancer, $\beta$ catenin mutations and abnormal protein expressions have been known in numerous human malignancies [6]. In human, breast cancer showed nuclear accumulation of $\beta$ catenin and stimulation of cyclin D1 expression, suggested that canonical wnt/ $\beta$-catenin signaling activated. In other cancers, the activation of canonical $\beta$-catenin/wnt signaling linked with the mutations of AXIN1, CTNNB1 or APC. However, these mutations are absent or rare in breast cancer [7].

For the inhibition of aberrant wnts, a scrutinized group of compounds has been reported as Inhibitors Wnt Proteins (IWPs) [8]. IWPs can inhibit the activity of wnt by altering other wnt signaling cascade members or by targeting RNA interferences [9]. The antagonists packed in liposome were the first designed inhibitors to directly inhibit the aberrant expression of mouse Wnt-3a. The interaction patterns of analyzed antagonists are still unknown. It advocates from the literature that they may have interacted with acyl compounds for the inhibition of possible wntfrizzled interactions. It is still vague about the binding domain and interacting residues of wnts. A hypothesis appeared from above logical arguments that the screened compounds from the pipeline can be used against wnt-8b for the identification of effective inhibitors from the reported library and also the interaction pattern. In silico approaches and bioinformatics analyses have demonstrated success in research methodologies directed at solving some biological problems [10]. They have also been used to design numerous novel computer-aided molecules against neurological disorders [11-15] and cancer [16-18]. In this current studies, we revealed direct inhibitors of acylated wnt-8b by employing in silico approaches followed by comparative modeling and comparative molecular docking studies.

\section{EXPERIMENTAL}

The amino acid (351 residues) sequence of wnt$8 b$ was retrieved in FASTA format from UniProt Knowledgebase (UniProtKB) having accession number Q93098. In present work, sequence analyses, 3D structure prediction and comparative molecular docking studies were performed.

\section{Structure prediction}

The amino acid sequence of Wnt-8b was retrieved from UniProtKB and was subjected to BLASTp for the identifications of suitable templates against Protein Data Bank (PDB). The utilized parameters were adjusted according to the nature of the query and the alignment was executed by using compositional matrix adjust scoring system. The templates against wnt-8b were retrieved and analyzed with regard to evalue, identity and query coverage for structure modeling. Comparative modeling and threading based approaches were also employed to predict the 3D structure using MODELLER 9.15, ITasser, Modweb, EsyPred, 3D-JigSaw and SwissModel which relies on the basis of spatial restraints.

Evaluation tools were applied to evaluate the accuracy of the predicted structures. Rampage generated ramachandran plot distributed the residues into the favored, allowed and outlier regions while ERRAT evaluated the overall quality factor of the predicted structures. The selected structure was subjected to the energy minimization using UCSF Chimera 1.10 and 1000 steps of conjugate gradient and steepest descent methods were executed with the amber ff99SB forcefield.

\section{Comparative docking}

The comparative modeling and threading techniques were used to predict the $3 \mathrm{D}$ models 
of the wnt-8b which has not been reported yet in biological databases and literature. Threading approach was also employed to predict the 3D structure of the wnt-8b to minimize the error rate. A compound library comprising of 271 molecules against wnt-4 was reported in the literature [8]. These reported compounds were retrieved from literature and followed by the comparative docking analyses.

The geometry optimization and energy minimization of the selected molecules were performed by Chem3D Ultra and UCSF Chimera 1.10. The docking studies were conducted to conclude the interaction and principles of bonding between protein and the ligands. The comparative docking analyses were performed by using AutoDock-Vina, GOLD and AutoDock4. Palmitoleic acid and Ser-186 were made flexible to obtain the concurrence in the docking experiments. Receptor regions encompassing thumb index (Ser-186) and palmitoleic acid were defined as target binding sites. The goldscore and chemscore scoring functions were preferred to evaluate the binding energies of all possible conformations of the compounds with the receptor protein. The log files were used to sort and rank the compounds on the basis of their Gold fitness scores and the complex with the highest score was exported to analyze the binding interactions. The top docked complexes were analyzed and visualized by UCSF Chimera 1.10. The flow chart depicting the sequence used in current studies is shown in Figure 1.

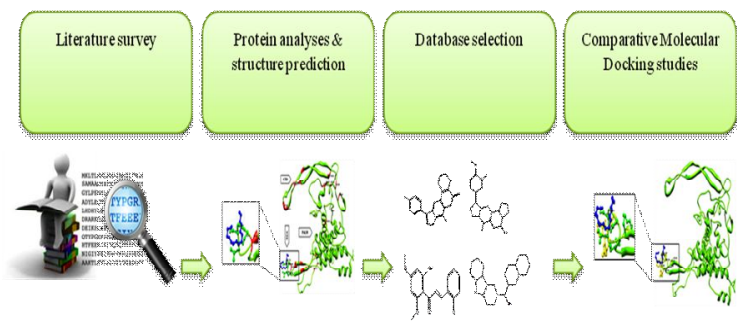

Figure 1: Flowchart scheme depicts the followed method

\section{RESULTS}

The current efforts summarize the inhibition of the aberrant expression of wnt-8b by utilizing the comparative molecular docking studies. Following analyses of the amino acid sequence of the wnt-8b using the information through NCBI and UniProtKB, the mature peptide had 24 cysteine residues (Figure 2) and two potentially $\mathrm{N}$-linked glycosylation sites that demonstrated the characteristic of the wnt-1 family signature motif; mature peptides of the human protein, wnt$8 \mathrm{~b}$, and wnt-8a have $66 \%$ identity. Also, it was observed that the initial 22 residues behaved like a signal peptide while the remaining 329 (23351) residues behaved like a mature peptide. The wnt-8b contained 24 residues of cysteine, 22 in mature peptide and two in signal peptide.

Number of Residues of Wnt-8b

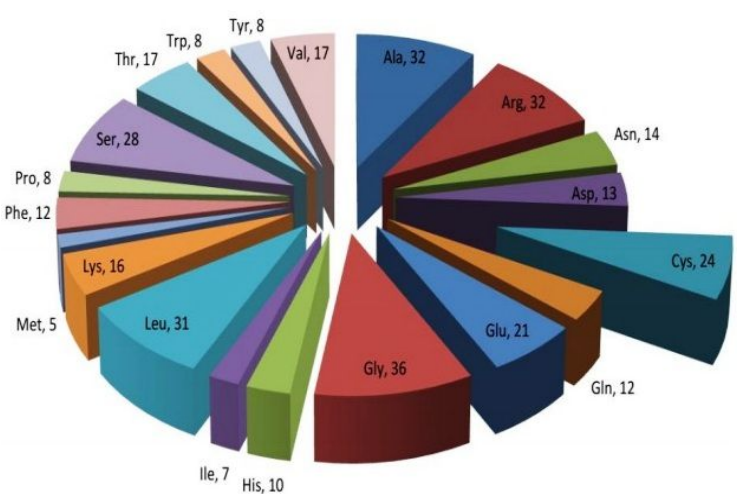

Figure 2: Distribution of the wnt-8b residues. The high composition of hydrophobic residues provides hydrophobic properties to wnt-8b. There are 24 Cysteine residues, i.e. a conserved property of the wnt family

In protparam analyses of the wnt-8b, $8.93 \mathrm{pl}$ value, extinction coefficient values at $280 \mathrm{~nm}$ ranged from 55920 to $57420 \mathrm{M}^{-1} \mathrm{~cm}^{-1}$, instability index of 54.43, grand average of hydrophobicity (GRAVY) value of -0.416 and 65.38 aliphatic index $(\mathrm{Al})$ value were observed.

Utilizing the suggested templates (Table 1) by the BLAST search to predict the 3D structures of the target protein, six templates (4FOA, 4KRR, 4OM8, 3TSR, 4M85 and 4MRU) emerged with optimal alignment sorted by e-value, query coverage, similarity and overall quality. The template, PDB ID: 4F0A, was observed as a suitable template with query sequence of $74 \%$ identity, $89 \%$ query coverage and $4 \mathrm{e}-178$ e-value with the target sequence.

Table 1: Templates for wnt-8b sorted by their query coverage and e-values

\begin{tabular}{cccccc}
\hline Template & $\begin{array}{c}\text { Max } \\
\text { score }\end{array}$ & $\begin{array}{c}\text { Total } \\
\text { score }\end{array}$ & $\begin{array}{c}\text { Query } \\
\text { coverage }\end{array}$ & $\begin{array}{c}\text { E- } \\
\text { value }\end{array}$ & $\begin{array}{c}\text { Identity } \\
(\%)\end{array}$ \\
\hline 4F0A & 501 & 501 & $89 \%$ & $4 \mathrm{e}-$ & 74 \\
& & & & 178 & \\
4KRR & 85.9 & 85.9 & $66 \%$ & $2 \mathrm{e}-19$ & 27 \\
4OM8 & 34.7 & 34.7 & $28 \%$ & 0.11 & 27 \\
3TSR & 29.6 & 29.6 & $9 \%$ & 4.2 & 42 \\
4M85 & 29.3 & 29.3 & $12 \%$ & 4.2 & 27 \\
4MRU & 28.9 & 28.9 & $29 \%$ & 8.7 & 22 \\
\hline
\end{tabular}

The structure prediction tools (3D-jigsaw, Swiss Model, I-TASSER and MODELLER 9.15) based on in silico approaches (homology modeling and 
threading) were utilized to predict 68 structures for wnt-8b. The predicted structures were evaluated on the basis of their favored regions, allowed regions, outliers, overall quality factor and structural patterns of the wnt family. Ramachandran plots of the predicted models presented the distribution of residues along with psi and phi distributions of non-glycine and nonproline residues. Psi and phi angles were plotted against each other to differentiate the outliers and the favored regions. The errat evaluation tool was utilized to calculate the overall quality factors of all the predicted structures by calculating the non-bonded interactions. Two lines were drawn on the error axis for the confidence to reject the regions that exceed the error values and the percentage of the protein for calculating the error value falls below the $95 \%$ of rejection limit. All the predicted models with their quality factors are presented in a single assessment plot. The most reliable structure was selected from the generated graph (Figure 3) having $82.456 \%$ overall quality factor and also compliance with the reported structural characteristics of wnt proteins.

The energy minimization applied to the selected structure of wnt-8b by utilizing UCSF Chimera 1.10 to examine its stereochemistry and minimize the energy constraints engaged the ff99SB forcefield with AMBER parameters and enhanced the backbone conformation of the predicted structure.

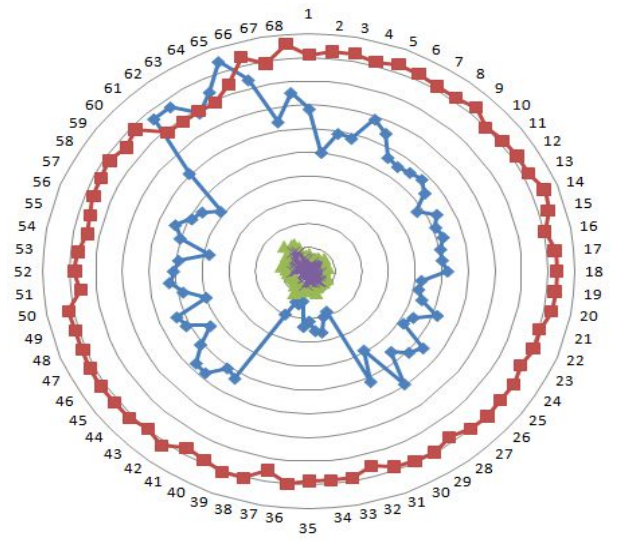

Figure 3: A comparative model assessment plot having the outliers, allowed regions, favored regions and the overall quality factor values

With the docking analyses performed against the wnt-8b covalently bounded with the palmitoleic acid to identify the maximum interaction affinity of the inhibitors, wnt-8b contained the $\mathrm{N}$-terminal domain (NTD) and C-terminal domain (CTD) separated by the Palm domain and the 24 cysteine residues considered as the significant residues (Figure 4).

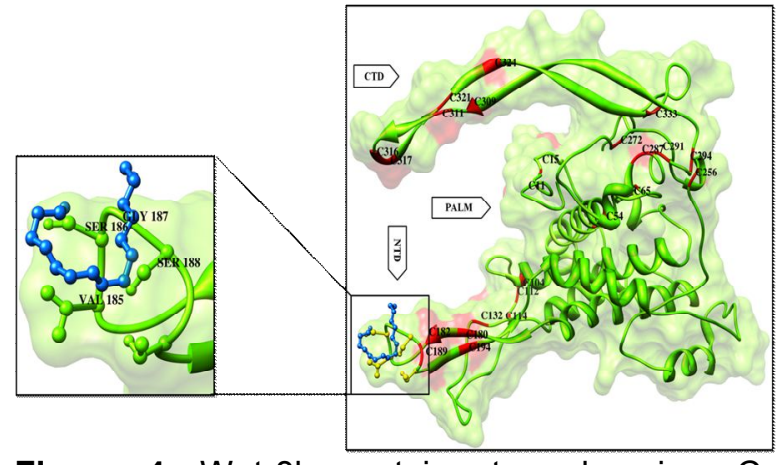

Figure 4: Wnt-8b contains two domains, Cterminal domain (CTD) and $\mathrm{N}$-terminal domain (NTD) which are separated by Palm domain, having 24 cysteine residues showed in red color

Comparative docking analyses of the selected library revealed variations in their binding energies. With the comparative docking analyses initially performed with 120 runs and all the generated docking complexes analyzed, suitable docked complex having lowest binding energy and highest binding affinity was chosen. The in silico analyses of the selected library were efficiently bound at the palmitoleic acid region of the protein. All the utilized docking tools showed effective binding energies and also exhibited the common binding domain.

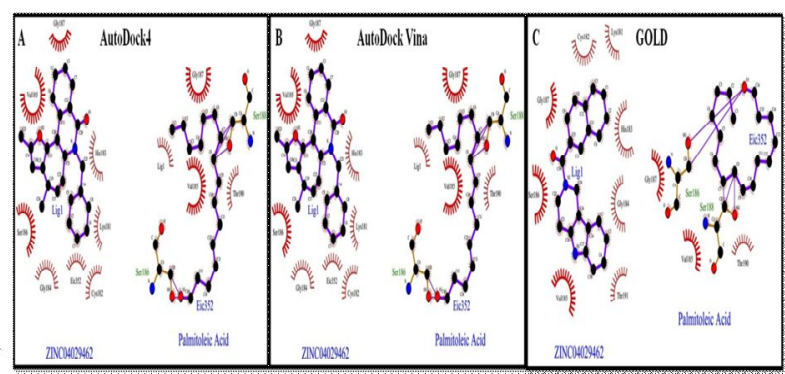

Figure 5: The inhibitor (ZINC04029462) showed interaction with palmitoleic acid and wnt-8b residues

It was observed that the ZINC04029462 compound interacted with wnt-8b and the palmitoleic acid and found common in all the utilized docking tools among top-ranked 20 compounds (Table 2). The common compound was selected on the basis of its highest binding affinity, gold fitness score and further evaluated on the basis of the interacting residues. Ligplot2 was used to analyze the interactions for the topranked 20 compounds from each docking tool and it was observed that the inhibitor bound with palmitoleic acid and wnt-8b (supplementary material 3). The interactions of ZINC04029462 from AutoDock-Vina, GOLD and Autodock4 was critically analyzed and visualized by ligplot2 (Figure 5). The interacting residues His-183, Val185, Ser-186, Gly-187, Ser-188 and Thr-190 were observed commonly among all the 
complexes and considered as the potential residues. When the interacting residues of all the docked complexes were analyzed utilizing the selected tools, it was observed that the selected common inhibitor (ZINC04029462) bound at the binding residues Val-185, Ser-186, Gly-187 and Ser-188 and also interacted with palmitoleic acid (Figure 6).

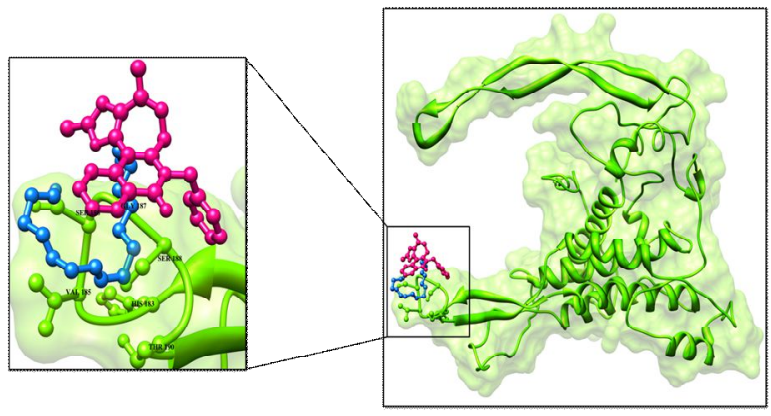

Figure 6: The interactions of the selected inhibitor (ZINC04029462) with the palmitoleic acid and wnt-8b

\section{DISCUSSION}

The extensive computational analyses of the structure of wnt-8b revealed two diverse domains; one is C-terminal domain (CTD) and the other one is N-terminal domain (NTD). The two domains (CTD and NTD) have resemblance with thumb and index finger connected by a hydrophobic region bordering on the palm [19]. The comparative analyses of the wnt-8b with crystal structure of the XWnt-8 revealed that Ser186 behaved as the acylation point for wnt-8b and also acylation as PTM. This acylation enables the wnt proteins to interact with frizzed receptors and enhances the wnt activity. The acylation inhibited by another inhibitor leads improper interactions of the wnt proteins with frizzled receptor or involved in the wnt signaling pathway. This acylation also increases the hydrophobicity of the wnt molecule [20].

By the structural analyses, it was analyzed that the twelve cysteine residues are involved in CTD, eight with NTD and the four cysteine residues are involved in the significant folding mechanism. The palmitoleic acid has an attachment at NTD and involved in the interaction between frizzled receptor and wnt-8b. The results suggest that the structural importance of the wnt-8b in wnt signaling activity. The computed $8.93 \mathrm{pl}$ value for wnt-8b predicts its basic behavior. The physiochemical properties of the wnt-8b were analyzed for the better understanding of the behavior and nature of the protein. The calculated $\mathrm{pl}$ will be helpful to develop the buffer system for the purification by isoelectric focusing method. The twenty-four cysteine residues were analyzed and considered as the characteristic feature of the wnt proteins. Wnt-8b was predicted as unstable on the calculated analyses of the instability index of 54.43 and grand GRAVY value of -0.416 .

The $65.38 \mathrm{Al}$ value defined the relative volume of the protein occupied by the aliphatic side chain and was regarded as the positive factor for the increase of the thermal stability of the proteins. The calculated Al of the wnt-8b depicts that it may not be stable for a wide range of temperatures.

Table 2: The top-ranked 20 compounds selected from AutoDockVina, GOLD and AutoDock4

\begin{tabular}{lccccc}
\hline Compound ID & $\begin{array}{c}\text { AutoDock } \\
\text { Vina B.E } \\
\text { (kcal/mol) }\end{array}$ & Compound ID & $\begin{array}{c}\text { GOLD } \\
\text { score }\end{array}$ & Compound ID & $\begin{array}{c}\text { AutoDock4 } \\
\text { B.E. } \\
\text { (kcal/mol) }\end{array}$ \\
\hline ZINC33834133 & -6.9 & ZINC02101579 & 43.27 & ZINC04029462 & -6.67 \\
ZINC02126329 & -6.7 & ZINC12296700 & 43.1 & ZINC00159463 & -5.83 \\
ZINC00892487 & -6.4 & ZINC03843242 & 43.1 & ZINC02101579 & -5.82 \\
ZINC02119405 & -6.3 & ZINC31167205 & 41.63 & ZINC08738236 & -5.78 \\
ZINC35442871 & -6.2 & ZINC00491326 & 41.24 & ZINC08738235 & -5.62 \\
ZINC14814670 & -6.2 & ZINC33831887 & 41.19 & ZINC04090656 & -5.62 \\
ZINC04029462 & -6.2 & ZINC00053074 & 41.08 & ZINC00095299 & -5.56 \\
ZINC03852034 & -6.2 & ZINC04029462 & $\mathbf{4 0 . 7 2}$ & ZINC02120567 & -5.51 \\
ZINC03852017 & -6.2 & ZINC02120567 & 40.63 & ZINC00095228 & -5.38 \\
ZINC03848414 & -6.2 & ZINC00491280 & 40.49 & ZINC02087427 & -5.36 \\
ZINC02121298 & -6.2 & ZINC02126390 & 40.43 & ZINC00634070 & -5.31 \\
ZINC02087427 & -6.2 & ZINC13410424 & 40.09 & ZINC00095171 & -5.31 \\
ZINC00489331 & -6.2 & ZINC02126329 & 40.07 & ZINC24716580 & -5.28 \\
ZINC00489027 & -6.2 & ZINC03843244 & 39.99 & ZINC02121298 & -5.28 \\
ZINC20112056 & -6.1 & ZINC04100773 & 39.98 & ZINC00622318 & -5.25 \\
ZINC13410424 & -6.1 & ZINC03852034 & 39.84 & ZINC00488772 & -5.22 \\
ZINC12296700 & -6.1 & ZINC13410456 & 39.31 & ZINC00095396 & -5.2 \\
ZINC03852015 & -6.1 & ZINC85876388 & 39.23 & ZINC00095284 & -5.2 \\
ZINC02149750 & -6.1 & ZINC03844502 & 39.2 & ZINC00095258 & -5.19 \\
ZINC02101408 & -6.1 & ZINC03843154 & 39.08 & ZINC00489027 & -5.17 \\
\hline
\end{tabular}


The 3D structure of the wnt-8b was not reported yet through NMR and X-ray crystallography techniques. The 3D structures lead to the insight understanding of the protein functions. Thus, the structure of wnt-8b was predicted by employing the comparative homology modeling and threading approaches. The optimally aligned top five suitable templates having the maximum identity, total scores, query coverage and evalues were used to predict the 3D structures for the wnt-8b. The overall query coverage among the utilized templates and target showed $89 \%$ from end to end that considered as satisfactory for the reliable structures by homology modeling approach. To overcome the errors and for better $3 \mathrm{D}$ structure, the threading approach was also utilized. The final selected structure showed 96.2 $\%$ residues in the favored and allowed regions which define the reliability of the structure for further analyses.

The extensive literature survey was conducted to scrutinize the appropriate library of 271 compounds against wnt-8b. However, the comparative docking studies of the selected library revealed fluctuations in their binding energies. The comparative docking analyses were performed and the generated complexes having the highest binding affinity and lowest binding energy was further analyzed. The elaborative in silico analyses of the selected compound library were efficiently bound at the similar binding region. Interestingly, it was also observed that all the compounds from the library showed effective binding at the same region of the protein and binding interactions depicts that the binding may have the ability to inhibit the aberrant expression of the wnt-8b. The comparative docking analyses also revealed that Val-185, Ser-186, Gly-187 and Ser-188 residues were effective for the receptor-ligand interactions. ZINC04029462 compound elucidated the efficient binding energy and common among all the utilized docking tools. It was observed that ZINC04029462 compound exhibited the binding interactions at the similar region of the protein where the remaining analyzed molecules bound. This compound showed more stability among all the analyzed compounds reflects better potential and may use to cure the aberrant expression of the wnt-8b.

\section{CONCLUSION}

The current analyses suggested that the ZINC04029462 compound efficiently binds with the target protein and also found common by analyzing through different docking tools. The comparative analyses proposed that the scrutinized compounds have better tendency to bind to the receptor protein efficiently. The interacting residues may also use for site mutagenesis studies and have the potential to lower down the aberrant expression of wnt-8b.

\section{DECLARATIONS}

\section{Acknowledgement}

The authors acknowledge Mr Tassaduq Hussain Jaffer, Bioinformatics Research Laboratory, Department of Biosciences, CIIT Sahiwal, for his support throughout the manuscript.

\section{Conflict of interest}

The authors declare no conflicts of interest in this work.

\section{Contribution of authors}

We declare that this work was done by the authors named in this article and all liabilities pertaining to claims relating to the content of this article will be borne by the authors.

\section{REFERENCES}

1. Janda CY, Waghray D, Levin AM, et al. (2012) Structural Basis of Wnt Recognition by Frizzled. Science (80- ) 337:59-64. doi: DOI 10.1126/science.1222879

2. Lako M, Strachan T, Curtis A, Lindsay S (1996) Isolation and characterization of WNT8B, a novel human Wnt gene that maps to 10q24. Genomics 35:386-388. doi: 10.1006/geno. 1996.0374

3. He X, Semenov M, Tamai K, Zeng $X$ (2004) LDL receptor-related proteins 5 and 6 in Wnt/beta-catenin signaling: arrows point the way. Development 131:1663-77. doi: 10.1242/dev.01117

4. Clements WM, Wang J, Sarnaik A, et al. (2002) $\beta$-catenin mutation is a frequent cause of Wnt pathway activation in gastric cancer. Cancer Res 62:3503-3506. doi: 10.1126/science. 275.5307.1790

5. Miao J, Kusafuka T, Kuroda S, et al. (2003) Mutation of $\beta$-catenin and its protein accumulation in solid and cystic tumor of the pancreas associated with metastasis. Int $\mathrm{J}$ Mol Med. doi: 10.3892/ijmm.11.4.461

6. Giles $R H$, Van Es JH, Clevers $H$ (2003) Caught up in a Wnt storm: Wnt signaling in cancer. Biochim Biophys Acta - Rev Cancer 1653:1-24. doi: 10.1016/S0304419X(03)00005-2

7. Benhaj K, Akcali KC, Ozturk M (2006) Redundant expression of canonical Wnt ligands in human breast cancer cell lines. Oncol Rep 15:701-707.

8. Hammad MA, Azam SS (2015) Structural dynamics and inhibitor searching for Wnt-4 protein using comparative computational studies. Drug Des Devel Ther 9:2449. 
9. Lewicki J, Axelrod F, Beviglia L, et al. (2012) Development of a novel Wnt pathway antagonist antibody, OMP-18R5, that reduces tumor initiating cell frequency in breast cancer. Cancer Res 72:3356.

10. Sehgal S, Khattak N, Mir A (2013) Structural, phylogenetic and docking studies of D-amino acid oxidase activator (DAOA), a candidate schizophrenia gene. Theor Biol Med Model 10:3. doi: 10.1186/17424682-10-3

11. Sehgal SA, Mannan S, Kanwal S, et al. (2015) Adaptive evolution and elucidating the potential inhibitor against schizophrenia to target DAOA (G72) isoforms. Drug Des Devel Ther 9:3471.

12. Sehgal SA, Hassan M, Rashid S (2014) Pharmacoinformatics elucidation of potential drug targets against migraine to target ion channel protein KCNK18. Drug Des Devel Ther 8:571.

13. Sehgal SA, Mannan S, Ali S (2016) Pharmacoinformatic and molecular docking studies reveal potential novel antidepressants against neurodegenerative disorders by targeting HSPB8. Drug Des Devel Ther 10:1605.

14. Sehgal SA (2017) Pharmacoinformatics, Adaptive Evolution, and Elucidation of Six Novel Compounds for Schizophrenia Treatment by Targeting DAOA (G72) Isoforms. Biomed Res Int 2017:1-19. doi: 10.1155/2017/5925714
15. Sehgal SA (2017) Pharmacoinformatics and molecular docking studies reveal potential novel Proline Dehydrogenase (PRODH) compounds for Schizophrenia inhibition. Med Chem Res 26:314-326. doi: 10.1007/s00044-016-1752-2

16. Sehgal SA, Tahir RA, Shafique S, et al. (2014) Molecular Modeling and Docking Analysis of CYP1A1 Associated with Head and Neck Cancer to Explore its Binding Regions. J. Theor. Comput. Sci. 2014:

17. Tahir RA, Sehgal SA, Khattak NA, et al. (2013) Tumor necrosis factor receptor superfamily 10B (TNFRSF10B): an insight from structure modeling to virtual screening for designing drug against head and neck cancer. Theor Biol Med Model 10:1

18. Kanwal S, Jamil F, Ali A, Sehgal SA (2016) Comparative Modeling, Molecular Docking, and Revealing of Potential Binding Pockets of RASSF2; a Candidate Cancer Gene. Interdiscip Sci Comput Life Sci 1-10.

19. Azam SS, Mirza AH (2014) Role of thumb index fold in Wnt-4 protein and its dynamics through a molecular dynamics simulation study. J Mol Liq 198:313-321. doi: http://dx.doi.org/10.1016/j.molliq.2014.07.007

20. Takada R, Satomi $Y$, Kurata $T$, et al. (2006) Monounsaturated fatty acid modification of Wnt protein: Its role in Wht secretion. Dev Cell 11:791-801. doi: DOI 10.1016/j.devcel.2006.10.003 